\title{
Article \\ Preparation of Thioaminals in Water
}

\author{
Lídia A. S. Cavaca, Rafael F. A. Gomes * and Carlos A. M. Afonso *
}

\begin{abstract}
Research Institute for Medicines (iMed.ULisboa), Faculty of Pharmacy, University of Lisbon, Av. Prof. Gama Pinto, 1649-003 Lisboa, Portugal; 1.cavaca@campus.fct.unl.pt

* Correspondence: rafael.gomes@campus.ul.pt (R.F.A.G.); carlosafonso@ff.ulisboa.pt (C.A.M.A.)
\end{abstract}

\begin{abstract}
The presence of sulfur-carbon bonds is transversal to several areas of chemistry, e.g., drug discovery, materials, and chemical biology. However, a lack of efficient and sustainable procedures for the preparation of thioaminals, the $\mathrm{N}, \mathrm{S}$-analogues of $\mathrm{O}, \mathrm{O}$-acetals, contributes to this functional group often being overlooked by the scientific community. In this work is described the formation of thioaminals in water promoted by copper(II) triflate.
\end{abstract}

Keywords: sustainability; aldehyde; catalysis

\section{Introduction}

The preparation of sulfur-rich frameworks has been a relevant topic for organic chemists, given its importance in materials $[1-4]$ and medicinal chemistry $[5,6]$. The need for the formation of C-S bonds led to developments such as the click thio-ene reaction, a cornerstone of material chemistry [1]. Additionally, thio-Michael additions are often used for the derivatization of activated olefins, a strategy widely used for the functionalization of materials and peptides [6,7].

Focusing on the medicinal chemistry area, sulfur is a key constituent of over $20 \%$ of FDA-approved drugs $[8,9]$, being responsible for favorable interactions on the targeted active site (Figure 1A) [10]. Amongst the sulfur-containing scaffolds, heterocycles such as thiazolidines and thiazolidinones have revealed a variety of biological activities [11-13], leading to abundant procedures for the formation of these cyclic $S, N$-aminals (thioaminals) [14-19]. However, reports on the formation of acyclic thioaminals are scarce, often requiring harsh conditions including hazardous reagents, affording poor yields or being limited to non-isolated intermediaries (Figure 1B) [20-23].

Recently, there has been an increased concern about the development of sustainable methodologies [24-27], including procedures in water [28-32]. With this in mind, our group (i) focused on the valorization of raw renewable materials such as furfural and hydroxymethylfurfural (HMF) [33-35] and (ii) reported on the use of water as a green reaction media for the production of cyclopentenones [36] and, more recently, aminals from the condensation of aryl aldehydes with amines [37]. This addresses one of the 12 principles of green chemistry [24], namely the use of less hazardous/toxic chemicals. The structural resemblance of aminals and thioaminals led us to enquire if a similar methodology could be applied to their formation. Herein is described the formation of thioaminals under aqueous conditions promoted by copper(II) triflate (Figure 1C).

Copyright: (ㅇ 2022 by the authors. Licensee MDPI, Basel, Switzerland. This article is an open access article distributed under the terms and conditions of the Creative Commons Attribution (CC BY) license (https:// creativecommons.org/licenses/by/ $4.0 /)$. 
A. Examples of FDA approved drugs containing Sulfur:<smiles>[R]O[Si]([O-])([O-])[O-]</smiles><smiles>Cc1ccc2c(c1)N(CCCN1CCNCC1)c1ccccc1S2</smiles><smiles>CC1(C)S[C@@H]2[C@H](NC(=O)[C@@H](N)c3ccc(O)cc3)C(=O)N2[C@H]1C(=O)O</smiles><smiles>CN(CCOc1ccc(CC2SC(=O)NC2=O)cc1)c1ccccn1</smiles>

Rosiglitazone anti-diabetes<smiles>Nc1nc2ccc(OC(F)(F)F)cc2s1</smiles>

B. Examples of previous methodologies aiming at preparing thioaminals:

Hajra and coworkers, 2019:<smiles>[CH2+]C(=O)c1ccc(OC)cc1</smiles>

Alper and coworkers, 1994:

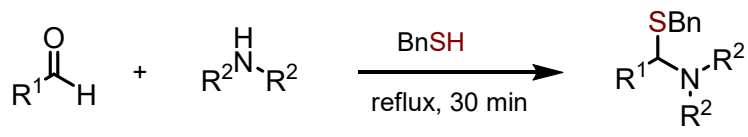

$$
\begin{aligned}
& 68-98 \%
\end{aligned}
$$

Katritzky and coworkers, 1986:

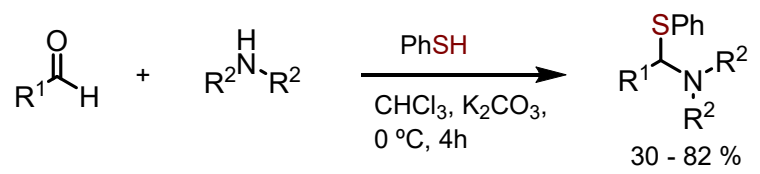

C. This work:

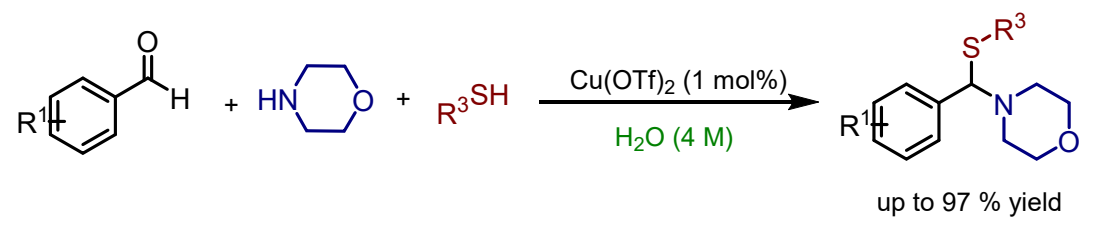

Figure 1. Importance of thiol in drug development (A); selected examples for the preparation of thioaminals (B); [20-23] this work (C).

\section{Results}

Selecting furfural, morpholine and thiophenol as model substrates, a screening of conditions was initially performed to identify the most suitable catalyst and solvent (Table 1). The formation of thioaminal 1 in the presence of catalytic copper(II) triflate (1 mol\%) in aqueous media afforded the desired product in $84 \%$ isolated yield after 10 min of reaction (Table 1, entry 3). In the absence of catalyst, $100 \%$ of starting furfural was recovered (Table 1, entry 1), while an increase in catalyst loading to $10 \mathrm{~mol} \%$ resulted in full conversion to the corresponding cyclopentenone (Table 1, entry 2) [36,38]. Other attempted metal-based catalysts (i.e., $\mathrm{CuSO}_{4}, \mathrm{FeCl}_{3}, \mathrm{AlCl}_{3}$ and $\mathrm{ZnCl}_{2}$ ) and Brønsted acids (i.e., triflic acid) also led to the formation of cyclopentenone as a side-product (Table 1, entries 7-11) [36,38]. Performing the reaction under neat conditions or by changing the solvent to ethanol or acetonitrile did not allow full conversion and caused a visible contamination 
with cyclopentenone (Table 1, entries 4-6). Thus, water was considered the most suitable solvent for this reaction.

Table 1. Effect of $\mathrm{Cu}(\mathrm{OTf})_{2}$ on the formation of thioaminals in aqueous media. ${ }^{\mathrm{a}}$ isolated yield. ${ }^{\mathrm{b}}$ Yield based on crude NMR.

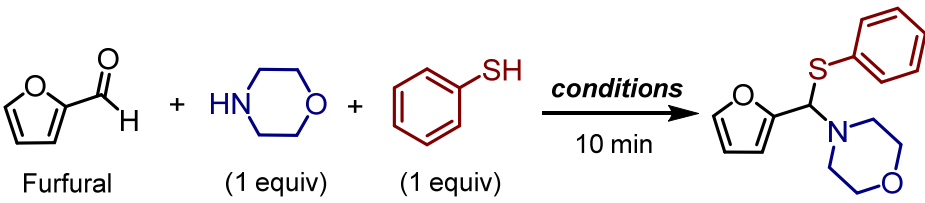

1

\begin{tabular}{ccccc}
\hline Entry & Catalyst & Solvent & Conversion (\%) & Yield of 1 (\%) \\
\hline 1 & no catalyst & $\mathrm{H}_{2} \mathrm{O}$ & 0 & 0 \\
2 & $\mathrm{Cu}(\mathrm{OTf})_{2}(10 \mathrm{~mol} \%)$ & $\mathrm{H}_{2} \mathrm{O}$ & 100 & 0 \\
$\mathbf{3}$ & $\mathrm{Cu}(\mathbf{O T f})_{2}(\mathbf{1 ~ m o l} \%)$ & $\mathbf{H}_{\mathbf{2}} \mathrm{O}$ & $\mathbf{1 0 0}$ & $\mathbf{1 0 0}(\mathbf{8 4})^{\mathrm{a}}$ \\
4 & $\mathrm{Cu}(\mathrm{OTf})_{2}(1 \mathrm{~mol} \%)$ & neat & 93 & $66^{\mathrm{a}, \mathrm{b}}$ \\
5 & $\mathrm{Cu}(\mathrm{OTf})_{2}(1 \mathrm{~mol} \%)$ & $\mathrm{EtOH}$ & 64 & $29^{\mathrm{a}, \mathrm{b}}$ \\
6 & $\mathrm{Cu}(\mathrm{OTf})_{2}(1 \mathrm{~mol} \%)$ & $\mathrm{MeCN}$ & 93 & $56^{\mathrm{a}, \mathrm{b}}$ \\
7 & $\mathrm{CuSO}(1 \mathrm{~mol} \%)$ & $\mathrm{H}_{2} \mathrm{O}$ & 100 & 35 \\
8 & $\mathrm{FeCl}_{3}(1 \mathrm{~mol} \%)$ & $\mathrm{H}_{2} \mathrm{O}$ & 100 & 0 \\
9 & $\mathrm{AlCl}_{3}(1 \mathrm{~mol} \%)$ & $\mathrm{H}_{2} \mathrm{O}$ & 100 & 12 \\
10 & $\mathrm{ZnCl}_{2}(1 \mathrm{~mol} \%)$ & $\mathrm{H}_{2} \mathrm{O}$ & 100 & 70 \\
11 & $\left.\mathrm{TfOH}^{2} \mathrm{~mol} \%\right)$ & $\mathrm{H}_{2} \mathrm{O}$ & 87 & 40 \\
\hline
\end{tabular}

Following the best selected conditions, we expanded the scope to a plethora of aryl and alkyl aldehydes (Scheme 1). First, we reacted benzaldehyde, morpholine and thiophenol to prepare thioaminal 2 in $76 \%$ yield. Electron-rich aromatic aldehydes, such as $4-\mathrm{NMe}_{2}-$ benzaldehyde and 4-OMe-benzaldehyde, resulted in no reaction or incomplete reaction (65\% conversion), and were impossible to isolate due to the product instability. Similar results were obtained when alkyl aldehydes were employed, resulting in a complex mixture.

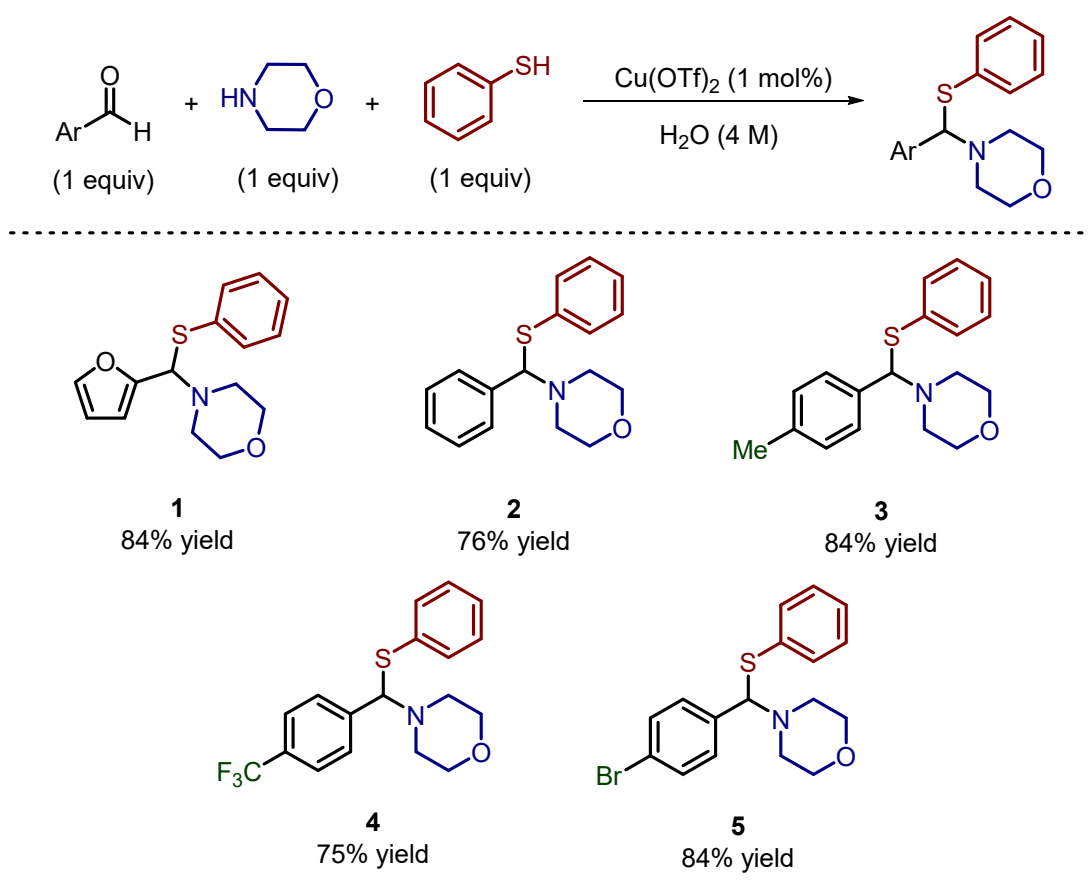

Scheme 1. Scope of aryl aldehydes for the formation of thioaminals. 
Counterintuitively, 4-Me-benzaldehyde afforded a better yield than 4- $\mathrm{CF}_{3}$-benzaldehyde, and a similar yield was obtained for the 4-Br-benzaldehyde derivative. Being electron poor, the formation of $\mathbf{4}$ and $\mathbf{5}$ should be favored since the aldehyde is more prone to undergo condensation with the secondary amine. Moreover, the use of 4- $\mathrm{NO}_{2}$-benzaldehyde only gave a $50 \%$ conversion to the corresponding thioaminal, without the possibility of purification. These unexpected results led us to conclude that other factors such as the stability of the constructs may play a role when analyzing the success of these reactions.

To our surprise, we did not observe the formation of the $N, N$ aminal product in this aldehyde scope, which clearly indicates that the $N, S$ thioaminals are thermodynamically more favorable. However, if we allowed for the reaction to proceed for longer periods of time, the yields would drop significantly. In this situation, no aldehyde is observed as well, indicating another decomposition pathway than the simple hydrolysis of the thioaminal. Focusing on isolation, we stumbled upon a major challenge, which was the hydrolysis of the thioaminals. Simple operations such as extraction, even at higher $\mathrm{pH}$ using $\mathrm{NaHCO}_{3}$ (sat aq.), led to hydrolysis and subsequently low yields of the products. Freeze-drying the solution, followed by washing the corresponding powders with cold water, significantly increased the isolated yields. Contrary to other prepared thioaminals, product 1, obtained from furfural, exhibited increased stability even during the work-up extraction with MTBE.

A search for secondary aliphatic and aromatic amines was also performed (Scheme 2). In addition to morpholine, other aliphatic amines such as piperidine, $N$-methyl piperazine and dibenzyl amine were tolerated under the reaction conditions, affording the corresponding thioaminals 6-8 in a good to excellent yield. These compounds exhibited hygroscopic properties, resulting in fast hydrolysis at room temperature or at $5{ }^{\circ} \mathrm{C}$. Bulky aliphatic amines, such as di-isopropylamine, did not react and the reagents were fully recovered. Surprisingly, diethylamine was also not a successful amine. An aromatic amine, $N$-methyl aniline, was employed under the reaction conditions, but no reaction occurred.

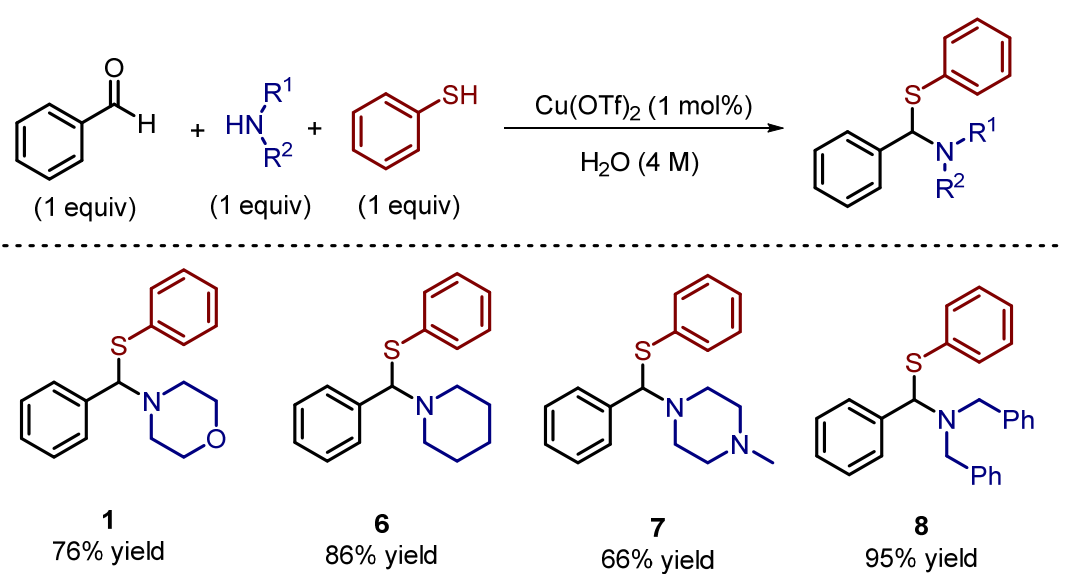

Scheme 2. Scope of alkyl amines for the formation of thioaminals.

Next, a series of aryl and aliphatic thiols were reacted with benzaldehyde and morpholine (Scheme 3). The reaction withstood electron-rich thiols such as 4-Me, 3,5-diMe, 4-OMe and 3,4-diOMe thiophenol yielding the thioaminal products 9-12 in good yield. Electron-poor thiol such as 4-F, 4-Cl and 3,5-diCl thiophenol also afforded the desired products, 13-15, in good to excellent yields. A reaction with 4- $\mathrm{NO}_{2}$-thiophenol was also attempted, but only $63 \%$ conversion to the thioaminal was observed, without the possibility of purification. Finally, alkyl thiols also afforded products 16-19 in good yield, even when long-alkyl-chain thiols (i.e., dodecanethiol) were employed. 


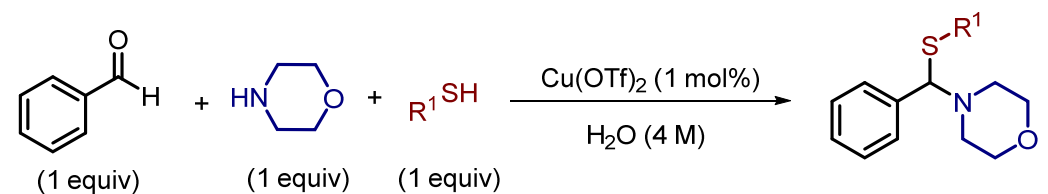<smiles>c1ccc(SC(c2ccccc2)N2CCOCC2)cc1</smiles>

2 $76 \%$ yield<smiles>COc1ccc(SC(c2ccccc2)N2CCOCC2)cc1OC</smiles>

12

$58 \%$ yield<smiles>c1ccc(CSC(c2ccccc2)N2CCOCC2)cc1</smiles>

16

$79 \%$ yield<smiles>[M]c1ccc(SC(c2ccccc2)N2CCOCC2)cc1</smiles>

9

$75 \%$ yield<smiles>Fc1ccc(SC(c2ccccc2)N2CCOCC2)cc1</smiles>

13

$70 \%$ yield<smiles>c1ccc(CCSC(c2ccccc2)N2CCOCC2)cc1</smiles>

17

90\% yield<smiles>Cc1cc(C)cc(SC(c2ccccc2)N2CCOCC2)c1</smiles>

10

$67 \%$ yield<smiles>Clc1ccc(SC(c2ccccc2)N2CCOCC2)cc1</smiles>

14

$74 \%$ yield<smiles>CCCSC(c1ccccc1)N1CCOCC1</smiles>

18

$71 \%$ yield<smiles>COc1ccc(SC(c2ccccc2)N2CCOCC2)cc1</smiles>

11

$86 \%$ yield
15

91\% yield<smiles>CC(C)CSC(c1ccccc1)N1CCOCC1</smiles>

Scheme 3. Scope of aryl and alkyl thiols for the formation of thioaminals.

An additional reaction using three solid substrates (i.e., 4-Br-benzaldehyde, 4-Clthiophenol and $\mathrm{N}$-acetylated piperazine) was performed in water. The corresponding thiominal was formed to some extent ( $68 \%$ conversion), indicating that solid substrates are more challenging and that the use of organic solvents might be necessary.

Intrigued by the selective formation of the thioaminal, a solution of the aminal in acetonitrile was reacted with thiophenol in the presence of a catalyst and a full conversion of aminal 20 was observed to the corresponding thioaminal 2, isolated in 94\% yield (Scheme 4).<smiles>c1ccc(C(N2CCOCC2)N2CCOCC2)cc1</smiles>

20

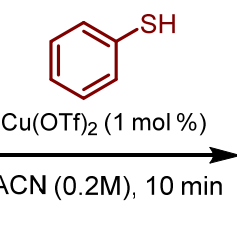

$\longrightarrow \mathrm{min}$<smiles>c1ccc(SC(c2ccccc2)N2CCOCC2)cc1</smiles>

$94 \%$ yield

Scheme 4. Amine/thiol exchange of aminals.

The proposed mechanism for the formation of the thioaminals depicted in Figure 2 is initiated by the formation of an iminium ion, which may either undergo further condensation with an amine (path a) or with a thiol (path b) to form the desired product. The fact that aminals in the presence of thiol lead to the formation of thioaminals suggests that even when the aminal is formed, its reversibility by the elimination of an amine generates the parent iminium ion, which will react with the thiol to form the product thioaminal. 


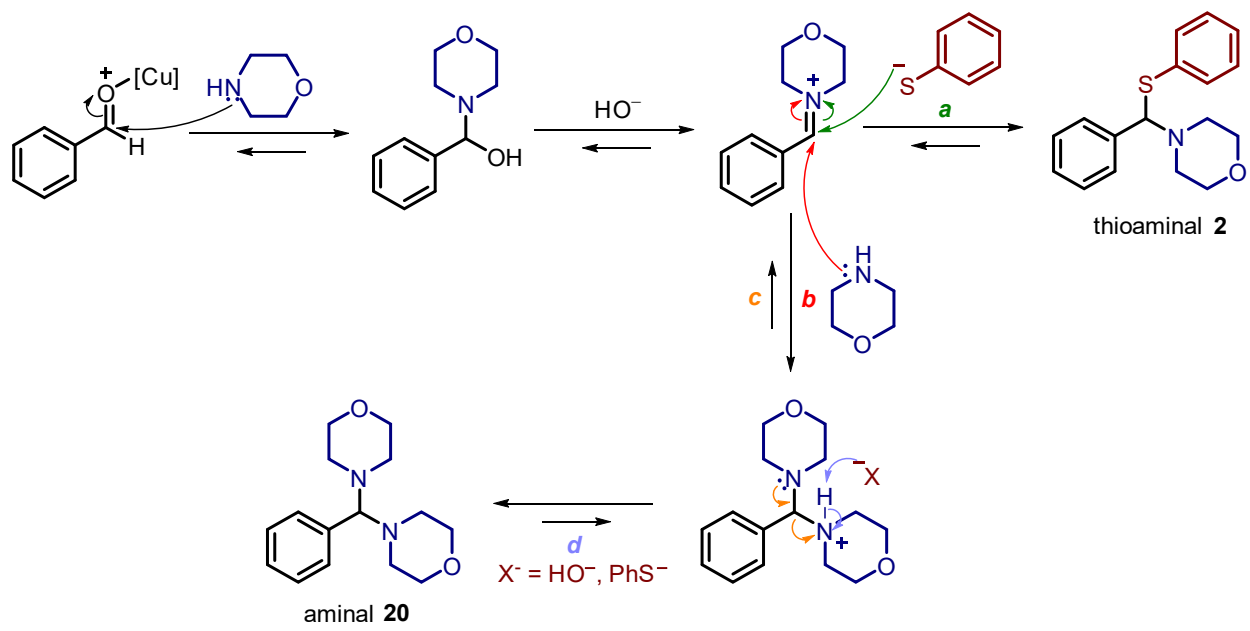

Figure 2. Proposed mechanism for the formation of thioaminals.

Following our previous studies on the stability of $\mathrm{N}, \mathrm{N}$-aminals, in which was observed that the electron-donating groups in the aldehyde stabilized the products, we enquired into the role of substituents in the arylthiol. To this end, we accessed the rates of hydrolysis of three thioaminals bearing electron-withdrawing $(\mathbf{1 4})$ and electron-donating $(\mathbf{9 , 1 1})$ para substituents by UV-vis spectroscopy, as depicted in Figure 3. We observed a fast hydrolysis of $<20 \mathrm{~s}$ in these diluted conditions (see Supplementary Materials, Figures S21 and S23). Despite this, a trend was observed where electron-withdrawing substituents on the thiol slightly hindered the hydrolysis of the products.

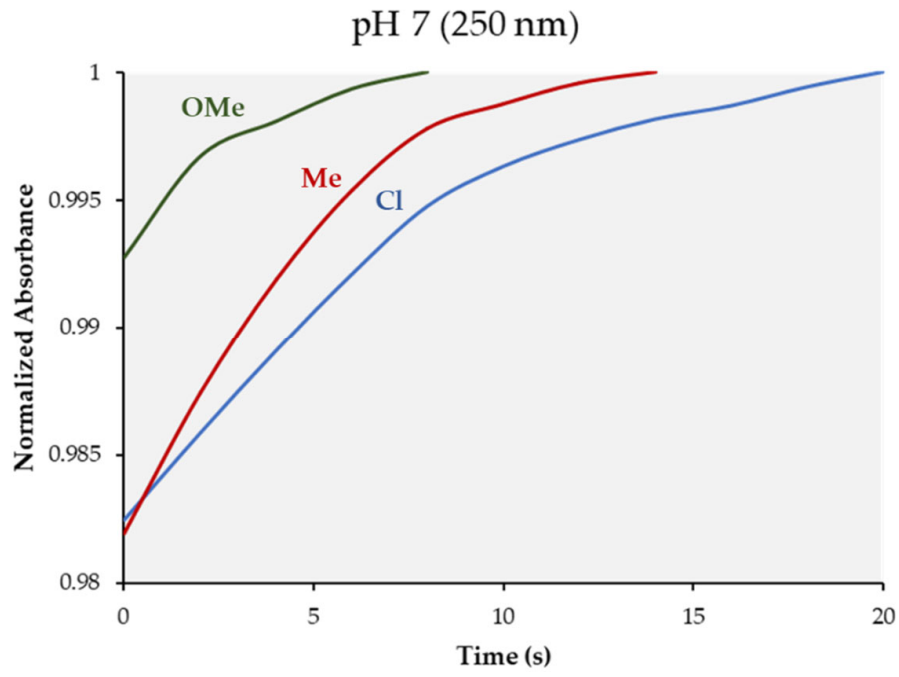

Figure 3. Hydrolysis evaluation by UV-vis spectroscopy of thioaminals 9, 11 and 14.

\section{Materials and Methods}

\subsection{General Information}

All solvents were of analytical grade and distilled prior to use. All reagents were used as received from commercial suppliers. NMR spectra were recorded in a Bruker Fourier 300 or Bruker Advance 400 spectrometer. High-Resolution Mass Spectrometry (HRMS) results were recorded in a Thermo Scientific $Q$ Exactive hybrid quadrupole-Orbitrap mass spectrometer (Thermo Scientific ${ }^{\mathrm{TM}} \mathrm{Q}$ Exactive ${ }^{\mathrm{TM}}$ Plus, Waltham, Massachusetts, U.S.). ${ }^{1} \mathrm{H} N M R,{ }^{13} \mathrm{C}$ NMR, HRMS and melting-point data are reported for all new compounds. The characterizations of reported compounds are in accordance with the literature. The UV 
stability measurements were traced in a Thermo Scientific ${ }^{\mathrm{TM}}$ Evolution $^{\mathrm{TM}} 201 \mathrm{UV}$-visible spectrophotometer (Thermo Scientific, Waltham, Massachusetts, U.S.).

\subsection{General Procedure for Cu(II)-Catalyzed Preparation of Thioaminals in Water}

Aldehyde (0.942 mmol, 1 equiv) and morpholine ( $0.942 \mathrm{mmol}, 1$ equiv) were placed in a round-bottom flask followed by addition of aqueous stock solution of $\mathrm{Cu}(\mathrm{OTf})_{2}(1$ $\mathrm{mol} \%, 227 \mu \mathrm{L}$ from $15 \mathrm{mg} / \mathrm{mL}$ stock solution). Then, thiol (0.942 mmol, 1 equiv) was added. The reaction was stirred at room temperature for $10 \mathrm{~min}$ and water removed by lyophilization to obtain pure thioaminals. Additional purification might have been required by washing with distilled water ( $\mathrm{pH}$ 9-10). In the case of thioaminal 1, an extraction with MTBE was performed.

\subsection{Characterization Data for Thioaminals}

4-(furan-2-yl(phenylthio)methyl)morpholine (1). Prepared according to general procedure using furfural (0.0905 g, $0.942 \mathrm{mmol}, 1$ equiv), morpholine (0.082 g, $0.942 \mathrm{mmol}, 1 \mathrm{equiv})$ and thiophenol $(0.1038 \mathrm{~g}, 0.942 \mathrm{mmol}, 1$ equiv $)$. The compound was obtained as a yellow solid $(0.2179 \mathrm{~g}, 84 \%$ yield) after extraction with MTBE ( $3 \mathrm{~mL}) .{ }^{1} \mathrm{H}$ NMR $(400 \mathrm{MHz}$, Acetone$\left.d_{6}\right): \delta 7.56-7.53(\mathrm{~m}, 1 \mathrm{H}), 7.50(\mathrm{dd}, J=7.2,1.8 \mathrm{~Hz}, 2 \mathrm{H}), 7.35-7.22(\mathrm{~m}, 3 \mathrm{H}), 6.52(\mathrm{~d}, J=3.3$ $\mathrm{Hz}, 1 \mathrm{H}), 6.40(\mathrm{dd}, J=3.3,1.9 \mathrm{~Hz}, 1 \mathrm{H}), 5.45(\mathrm{~s}, 1 \mathrm{H}), 3.59(\mathrm{q}, J=5.3 \mathrm{~Hz}, 4 \mathrm{H}), 2.78-2.62(\mathrm{~m}$, $4 \mathrm{H}) \mathrm{ppm} .{ }^{13} \mathrm{C}$ NMR $\left(101 \mathrm{MHz}\right.$, Acetone- $\left.d_{6}\right): \delta 152.9,143.5,136.9,133.2,129.8,127.8,111.0$, $110.5,76.2,67.2,50.0$ ppm. m.p. $=50-52{ }^{\circ} \mathrm{C}$. HRMS $(\mathrm{m} / z)$ calcd for $\mathrm{C}_{15} \mathrm{H}_{17} \mathrm{NO}_{2} \mathrm{~S}\left([\mathrm{M}+\mathrm{H}]^{+}\right)$ 276.10528, found 276.10445.

4-(phenyl(phenylthio)methyl)morpholine (2). Prepared according to general procedure using benzaldehyde $(0.1054 \mathrm{~g}, 0.993 \mathrm{mmol}, 1$ equiv $)$, morpholine $(0.0887 \mathrm{~g}, 1.02 \mathrm{mmol}, 1.03$ equiv) and thiophenol ( $0.1084 \mathrm{~g}, 0.984 \mathrm{mmol}, 0.99$ equiv). The compound was obtained as a pale-yellow solid (0.2072 g, 76\% yield). ${ }^{1} \mathrm{H}$ NMR (300 MHz, Acetone- $\left.d_{6}\right): \delta 7.65-7.61$ $(\mathrm{m}, 2 \mathrm{H}), 7.47-7.44(\mathrm{~m}, 2 \mathrm{H}), 7.40-7.21(\mathrm{~m}, 6 \mathrm{H}), 5.53(\mathrm{~s}, 1 \mathrm{H}), 3.64-3.53(\mathrm{~m}, 4 \mathrm{H}), 2.75-2.60(\mathrm{~m}$, 4H) ppm [39]. ${ }^{13} \mathrm{C}$ NMR (75 MHz, Acetone- $\left.d_{6}\right): \delta 139.9,137.7,132.8,129.6,128.9,128.3$, 128.0, 127.5, 81.7, 67.3, 50.3. m.p. $=41-43{ }^{\circ} \mathrm{C}\left(\right.$ lit. $\left.35-36{ }^{\circ} \mathrm{C}\right)$ [40]. HRMS $(\mathrm{m} / \mathrm{z})$ calcd for $\mathrm{C}_{17} \mathrm{H}_{19} \mathrm{NOS}\left([\mathrm{M}+\mathrm{H}]^{+}\right)$286.12601, found 286.12766 .

4-((phenylthio)(p-tolyl)methyl)morpholine (3). Prepared according to general procedure using 4-methylbenzaldehyde $(0.1311 \mathrm{~g}, 1.09 \mathrm{mmol}, 1$ equiv), morpholine (0.11312 $\mathrm{g}, 1.3$ mmol, 1.19 equiv) and thiophenol ( $0.1038 \mathrm{~g}, 0.942 \mathrm{mmol}, 0.86 \mathrm{equiv})$. The compound was obtained as a pale-yellow solid $\left(0.2371 \mathrm{~g}, 84 \%\right.$ yield). ${ }^{1} \mathrm{H}$ NMR (300 MHz, Acetone- $\left.d_{6}\right)$ : $\delta$ 7.52-7.49 (m, 2H), 7.46-7.43 (m, 2H), 7.29-7.22 (m, 2H), 7.22-7.16 (m, 3H), $5.50(\mathrm{~s}, 1 \mathrm{H})$, 3.61-3.55 (m, 4H), 2.70-2.62 (m, 4H), $2.32(\mathrm{~s}, 3 \mathrm{H}) \mathrm{ppm} .{ }^{13} \mathrm{C}$ NMR (101 MHz, Acetone- $\left.d_{6}\right): \delta$ 139.2, 137.5, 135.7, 132.8, 130.2, 129.7, 129.7, 128.5, 128.2, 89.5, 82.9, 68.1, 67.6, 67.5, 67.3, 65.7, $51.1,50.4,50.2,48.0,46.9,21.0$ ppm. m.p. $=44-47^{\circ} \mathrm{C}$. HRMS $(\mathrm{m} / z)$ calcd for $\mathrm{C}_{18} \mathrm{H}_{21} \mathrm{NOS}$ $\left([\mathrm{M}+\mathrm{H}]^{+}\right) 300.14166$, found 300.14304 .

4-((phenylthio)(4-(trifluoromethyl)phenyl)methyl)morpholine (4). Prepared according to general procedure using 4-(trifluoromethyl)benzaldehyde ( $0.1634 \mathrm{~g}, 0.938 \mathrm{mmol}, 1$ equiv), morpholine ( $0.0835 \mathrm{~g}, 0.958 \mathrm{mmol}, 1.02$ equiv) and thiophenol $(0.1308 \mathrm{~g}, 1.19 \mathrm{mmol}, 1.27$ equiv). The compound was obtained as a pale-orange solid $(0.2489 \mathrm{~g}, 75 \%$ yield $) .{ }^{1} \mathrm{H}$ NMR (300 MHz, Acetone- $\left.d_{6}\right): \delta 7.91-7.87(\mathrm{~m}, 2 \mathrm{H}), 7.74-7.70(\mathrm{~m}, 2 \mathrm{H}), 7.50-7.46(\mathrm{~m}, 2 \mathrm{H})$, 7.36-7.21 (m, 3H), $5.62(\mathrm{~s}, 1 \mathrm{H}), 3.69-3.55(\mathrm{~m}, 4 \mathrm{H}), 2.77-2.63(\mathrm{~m}, 4 \mathrm{H}) \mathrm{ppm} .{ }^{13} \mathrm{C}$ NMR $(101$ $\mathrm{MHz}$, Acetone- $\left.d_{6}\right): \delta 144.7,136.9,133.0,130.4,130.0,129.2(\mathrm{q}), 127.9,127.2(\mathrm{q}), 125.86(\mathrm{q})$, 82.5, 67.2, 50.4 ppm. m.p. $=63-64{ }^{\circ} \mathrm{C}$. HRMS $(m / z)$ calcd for $\mathrm{C}_{18} \mathrm{H}_{18} \mathrm{~F}_{3} \mathrm{NOS}\left([\mathrm{M}+\mathrm{H}]^{+}\right)$ 354.11340 , found 354.11067.

4-((4-bromophenyl)(phenylthio)methyl)morpholine (5). Prepared according to general procedure using 4-bromobenzaldehyde $(0.1728 \mathrm{~g}, 0.934 \mathrm{mmol}, 1$ equiv), morpholine $(0.0821$ $\mathrm{g}, 0.942 \mathrm{mmol}, 1.01 \mathrm{equiv})$ and thiophenol ( $0.104 \mathrm{~g}, 0.942 \mathrm{mmol}, 1.01 \mathrm{equiv})$. The compound was obtained as a pale-yellow solid $(0.2843 \mathrm{~g}, 84 \%$ yield $) .{ }^{1} \mathrm{H}$ NMR $\left(400 \mathrm{MHz}\right.$, Acetone- $\left.d_{6}\right)$ : $\delta 7.61(\mathrm{~d}, J=8.5 \mathrm{~Hz}, 2 \mathrm{H}), 7.55(\mathrm{~d}, J=8.5 \mathrm{~Hz}, 3 \mathrm{H}), 7.47-7.43(\mathrm{~m}, 2 \mathrm{H}), 7.27(\mathrm{dd}, J=7.1,3.4$ $\mathrm{Hz}, 3 \mathrm{H}), 5.52(\mathrm{~s}, 1 \mathrm{H}), 3.65-3.54(\mathrm{~m}, 6 \mathrm{H}), 2.74-2.60(\mathrm{~m}, 4 \mathrm{H}) \mathrm{ppm} .{ }^{13} \mathrm{C}$ NMR $(101 \mathrm{MHz}$, 
Acetone- $\left.d_{6}\right): \delta 139.5,137.2,132.9,132.0,131.8,130.2,129.9,128.2,127.7,122.1,82.4,67.3,50.3$ ppm. m.p. $=58-60{ }^{\circ} \mathrm{C}$. HRMS $(m / z)$ calcd for $\mathrm{C}_{17} \mathrm{H}_{18} \operatorname{BrNOS}\left([\mathrm{M}+\mathrm{H}]^{+}\right) 364.03652$, found 364.03788.

1-(phenyl(phenylthio)methyl)piperidine (6). Prepared according to general procedure using benzaldehyde $(0.100 \mathrm{~g}, 0.972 \mathrm{mmol}, 1$ equiv $)$, piperidine $(0.0802 \mathrm{~g}, 0.942 \mathrm{mmol}, 1$ equiv) and thiophenol ( $0.1038 \mathrm{~g}, 0.942 \mathrm{mmol}, 1 \mathrm{equiv})$. The compound was obtained as a pale-yellow oil $\left(0.2307 \mathrm{~g}, 86 \%\right.$ yield). ${ }^{1} \mathrm{H}$ NMR $\left(300 \mathrm{MHz}\right.$, Acetone- $\left.d_{6}\right): \delta 7.69-7.63(\mathrm{~m}$, 2H), 7.48-7.41 (m, 2H), 7.38-7.21 (m, 7H), 5.57 (s, 1H), 2.65 (d, J = 17.4 Hz, 4H), 1.57-1.39 (m, 7H) ppm. ${ }^{13} \mathrm{C}$ NMR (101 MHz, Acetone- $\left.d_{6}\right): \delta$ 140.7, 137.3, 133.0, 132.4, 130.1, 129.7, 129.5, $128.7,128.6,128.1,84.5,50.9,26.6,25.0$ ppm. HRMS $(m / z)$ calcd for $\mathrm{C}_{18} \mathrm{H}_{21} \mathrm{NS}\left([\mathrm{M}+\mathrm{H}]^{+}\right)$ 284.14675, found 284.14743.

1-methyl-4-(phenyl(phenylthio)methyl)piperazine (7). Prepared according to general procedure using benzaldehyde $(0.100 \mathrm{~g}, 0.942 \mathrm{mmol}, 1$ equiv), $N$-methyl piperazine (0.0944 $\mathrm{g}, 0.942 \mathrm{mmol}, 1$ equiv) and thiophenol ( $0.1038 \mathrm{~g}, 0.942 \mathrm{mmol}, 1$ equiv). The compound was obtained as a hygroscopic orange solid $(0.1852 \mathrm{~g}, 66 \%$ yield $) .{ }^{1} \mathrm{H}$ NMR $(400 \mathrm{MHz}$, Acetone- $\left.d_{6}\right): \delta 7.67-7.61(\mathrm{~m}, 2 \mathrm{H}), 7.56-7.52(\mathrm{~m}, 1 \mathrm{H}), 7.47-7.42(\mathrm{~m}, 2 \mathrm{H}), 7.38-7.33(\mathrm{~m}, 3 \mathrm{H})$, $7.28(\mathrm{dt}, J=10.6,7.6 \mathrm{~Hz}, 4 \mathrm{H}), 5.59(\mathrm{~s}, 1 \mathrm{H}), 2.76-2.63(\mathrm{~m}, 4 \mathrm{H}), 2.34(\mathrm{~s}, 5 \mathrm{H}), 2.16(\mathrm{~s}, 3 \mathrm{H})$ ppm. ${ }^{13} \mathrm{C}$ NMR $\left(101 \mathrm{MHz}\right.$, Acetone- $\left.d_{6}\right): \delta 140.3,137.4,132.6,130.2,129.7,129.6,128.8,128.5$, $128.3,128.2,127.3,83.1,55.8,46.3$ ppm. m.p. = low melting point. HRMS $(m / z)$ calcd for $\mathrm{C}_{18} \mathrm{H}_{22} \mathrm{~N}_{2} \mathrm{~S}\left([\mathrm{M}+\mathrm{H}]^{+}\right)$299.15765, found 299.16097 .

$N, N$-dibenzyl-1-phenyl-1-(phenylthio)methanamine (8). Prepared according to general procedure using benzaldehyde $(0.100 \mathrm{~g}, 0.942 \mathrm{mmol}, 1$ equiv), dibenzylamine $(0.1859 \mathrm{~g}$, $0.942 \mathrm{mmol}, 1$ equiv) and thiophenol $(0.1038 \mathrm{~g}, 0.942 \mathrm{mmol}, 1 \mathrm{equiv})$. The compound was obtained as a pale-yellow oil $\left(0.3547 \mathrm{~g}, 95 \%\right.$ yield). ${ }^{1} \mathrm{H}$ NMR (300 MHz, Acetone- $\left.d_{6}\right): \delta$ 7.82-7.76 (m, 2H), 7.42-7.37 (m, 2H), 7.33-7.19 (m, 17H), $5.48(\mathrm{~s}, 1 \mathrm{H}), 3.79(\mathrm{~s}, 4 \mathrm{H}) \mathrm{ppm} .{ }^{13} \mathrm{C}$ NMR $\left(101 \mathrm{MHz}\right.$, Acetone- $\left.d_{6}\right): \delta 140.9,139.6,137.6,132.7,129.9,129.8,129.7,129.3,129.2$, $129.01,129.0,128.7,128.1,127.4,79.0,54.4$ ppm. HRMS $(m / z)$ calcd for $\mathrm{C}_{27} \mathrm{H}_{25} \mathrm{NS}\left([\mathrm{M}+\mathrm{H}]^{+}\right)$ 396.17805, found 396.17722.

4-(phenyl(p-tolylthio)methyl)morpholine (9). Prepared according to general procedure using benzaldehyde $(0.0997 \mathrm{~g}, 0.939 \mathrm{mmol}, 1$ equiv $)$, morpholine $(0.0833 \mathrm{~g}, 0.956 \mathrm{mmol}$, 1.02 equiv) and 4-methylbenzenethiol ( $0.1198 \mathrm{~g}, 0.965 \mathrm{mmol}, 1.03 \mathrm{equiv})$. The compound was obtained as a pale-yellow solid $(0.2097 \mathrm{~g}, 75 \%$ yield $) .{ }^{1} \mathrm{H}$ NMR (300 MHz, Acetone- $\left.d_{6}\right)$ : $\delta 7.63-7.60(\mathrm{~m}, 2 \mathrm{H}), 7.42-7.29(\mathrm{~m}, 5 \mathrm{H}), 7.09(\mathrm{~d}, J=7.8 \mathrm{~Hz}, 2 \mathrm{H}), 5.43(\mathrm{~s}, 1 \mathrm{H}), 3.61-3.56(\mathrm{~m}$, $4 \mathrm{H}), 2.72-2.62(\mathrm{~m}, 4 \mathrm{H}), 2.27(\mathrm{~s}, 3 \mathrm{H}) \mathrm{ppm} .{ }^{13} \mathrm{C}$ NMR $\left(101 \mathrm{MHz}\right.$, Acetone- $\left.d_{6}\right): \delta 140.0,137.5$, $133.9,133.3,130.5,129.6,128.9,128.5,83.5,67.3,50.3,21.0$ ppm. m.p. $=45-47^{\circ} \mathrm{C}$. HRMS $(\mathrm{m} / z)$ calcd for $\mathrm{C}_{18} \mathrm{H}_{21} \mathrm{NOS}\left([\mathrm{M}+\mathrm{H}]^{+}\right) 300.14166$, found 300.14249 .

4-(((3,5-dimethylphenyl)thio)(phenyl)methyl)morpholine (10). Prepared according to general procedure using benzaldehyde $(0.1008 \mathrm{~g}, 0.950 \mathrm{mmol}, 1 \mathrm{equiv})$, morpholine $(0.083$ g, $0.953 \mathrm{mmol}, 1$ equiv) and 3,5-dimethylbenzenethiol ( $0.1295 \mathrm{~g}, 0.937 \mathrm{mmol}, 0.99$ equiv). The compound was obtained as a pale-yellow solid $\left(0.190 \mathrm{~g}, 67 \%\right.$ yield). ${ }^{1} \mathrm{H}$ NMR (300 $\mathrm{MHz}$, Acetone- $\left.d_{6}\right): \delta 7.65-7.62(\mathrm{~m}, 2 \mathrm{H}), 7.39-7.27(\mathrm{~m}, 4 \mathrm{H}), 7.10-7.09(\mathrm{~m}, 2 \mathrm{H}), 5.52(\mathrm{~s}, 1 \mathrm{H})$, 3.69-3.53 (m, 4H), 2.74-2.59 (m, 4H), $2.22(\mathrm{~m}, 6 \mathrm{H})$ ppm. ${ }^{13} \mathrm{C}$ NMR (101 MHz, Acetone- $\left.d_{6}\right): \delta$ $140.1,139.1,137.3,130.2,129.6,129.2,128.9,125.8,82.9,67.3,50.3,21.2$ ppm. m.p. $=37-39$ ${ }^{\circ} \mathrm{C}$. HRMS $(m / z)$ calcd for $\mathrm{C}_{19} \mathrm{H}_{23} \mathrm{NOS}\left([\mathrm{M}+\mathrm{H}]^{+}\right)$314.15731, found 314.16031.

4-((4-methoxyphenyl)thio)(phenyl)methyl)morpholine (11). Prepared according to general procedure using benzaldehyde $(0.1014 \mathrm{~g}, 0.955 \mathrm{mmol}, 1 \mathrm{equiv})$, morpholine $(0.0939 \mathrm{~g}$, $1.08 \mathrm{mmol}, 1.13$ equiv) and 4-methoxybenzenethiol ( $0.1326 \mathrm{~g}, 0.946 \mathrm{mmol}, 0.99$ equiv) The compound was obtained as a pale-yellow solid $(0.2089 \mathrm{~g}, 86 \%$ yield $) .{ }^{1} \mathrm{H}$ NMR (300 $\mathrm{MHz}$, Acetone- $\left.d_{6}\right): \delta 7.59-7.57(\mathrm{~m}, 2 \mathrm{H}), 7.41-7.25(\mathrm{~m}, 5 \mathrm{H}), 6.87-6.84(\mathrm{~m}, 2 \mathrm{H}), 5.29(\mathrm{~s}, 1 \mathrm{H})$, $3.77(\mathrm{~s}, 3 \mathrm{H}), 3.65-3.52(\mathrm{~m}, 4 \mathrm{H}), 2.75-2.68(\mathrm{~m}, 2 \mathrm{H}), 2.65-2.59(\mathrm{~m}, 2 \mathrm{H}) \mathrm{ppm} .{ }^{13} \mathrm{C}$ NMR $(75$ $\mathrm{MHz}$, Acetone- $\left.d_{6}\right): \delta 160.3,140.1,135.9,129.6,128.8,128.5,127.4,115.4,84.1,67.4,55.6,50.5$ ppm. m.p. $=69-71^{\circ} \mathrm{C}$. HRMS $(m / z)$ calcd for $\mathrm{C}_{18} \mathrm{H}_{21} \mathrm{NO}_{2} \mathrm{~S}\left([\mathrm{M}+\mathrm{H}]^{+}\right) 316.13658$, found 316.13639 . 
4-(((3,4-dimethoxyphenyl)thio)(phenyl)methyl)morpholine (12). Prepared according to general procedure using benzaldehyde $(0.1003 \mathrm{~g}, 0.945 \mathrm{mmol}, 1$ equiv $)$, morpholine $(0.0853$ $\mathrm{g}, 0.979 \mathrm{mmol}, 1.04$ equiv) and 3,5-dimethoxybenzenethiol ( $0.159 \mathrm{~g}, 0.934 \mathrm{mmol}, 0.99 \mathrm{equiv})$. The compound was obtained as a pale-yellow solid $\left(0.187 \mathrm{~g}, 58 \%\right.$ yield). ${ }^{1} \mathrm{H}$ NMR (300 $\mathrm{MHz}$, Acetone- $\left.d_{6}\right): \delta 7.59-7.56(\mathrm{~m}, 2 \mathrm{H}), 7.38-7.28(\mathrm{~m}, 3 \mathrm{H}), 7.02-6.99(\mathrm{~m}, 2 \mathrm{H}), 6.86-6.83$ $(\mathrm{m}, 1 \mathrm{H}), 5.35(\mathrm{~s}, 1 \mathrm{H}), 3.77(\mathrm{~s}, 1 \mathrm{H}), 3.74(\mathrm{~s}, 3 \mathrm{H}), 3.62-3.57(\mathrm{~m}, 4 \mathrm{H}), 2.73-2.64(\mathrm{~m}, 4 \mathrm{H}) .{ }^{13} \mathrm{C}$ NMR (101 MHz, Acetone- $\left.d_{6}\right): \delta$ 206.2, 151.0, 150.6, 140.1, 130.3, 129.6, 128.8, 128.4, 126.9, $124.4,115.0,112.9,83.7,67.4,56.1,56.1,50.5$ ppm. m.p. $=74-78{ }^{\circ} \mathrm{C}$. HRMS $(\mathrm{m} / \mathrm{z})$ calcd for $\mathrm{C}_{19} \mathrm{H}_{23} \mathrm{NO}_{3} \mathrm{~S}\left([\mathrm{M}+\mathrm{H}]^{+}\right)$346.14714, found 346.14753.

4-((4-fluorophenyl)thio)(phenyl)methyl)morpholine (13). Prepared according to general procedure using benzaldehyde $(0.1066 \mathrm{~g}, 1.0 \mathrm{mmol}, 1 \mathrm{equiv})$, morpholine $(0.0916 \mathrm{~g}, 1.05$ mmol, 1.05 equiv) and 4-fluorobenzenethiol ( $0.1283 \mathrm{~g}, 1.0 \mathrm{mmol}, 1$ equiv). The compound was obtained as a pale-yellow solid $(0.2065 \mathrm{~g}, 70 \%$ yield $) .{ }^{1} \mathrm{H}$ NMR $\left(300 \mathrm{MHz}\right.$, Acetone- $\left.d_{6}\right)$ : $\delta$ 7.61-7.56 (m, 2H), 7.53-7.46 (m, 2H), 7.39-7.30 (m, 3H), 7.09-7.02 (t, J = 8.8 Hz, 2H), $5.44(\mathrm{~s}$, $1 \mathrm{H}), 3.61-3.56(\mathrm{~m}, 5 \mathrm{H}), 2.70-2.64(\mathrm{~m}, 4 \mathrm{H}) \mathrm{ppm} .{ }^{13} \mathrm{C}$ NMR $\left(101 \mathrm{MHz}\right.$, Acetone- $\left.d_{6}\right): \delta 164.7$, $162.3,139.5,132.9,132.1,132.0,129.6,128.9,128.5,117.3,117.1,83.6,67.5,50.4$ ppm. m.p. $=$ 47-49 ${ }^{\circ} \mathrm{C}$. HRMS $(m / z)$ calcd for $\mathrm{C}_{17} \mathrm{H}_{18}$ FNOS $\left([\mathrm{M}+\mathrm{H}]^{+}\right)$304.11659, found 304.11812.

4-((4-chlorophenyl)thio)(phenyl)methyl)morpholine (14). Prepared according to general procedure using benzaldehyde $(0.100 \mathrm{~g}, 0.942 \mathrm{mmol}, 1$ equiv), morpholine $(0.0869 \mathrm{~g}, 0.997$ mmol, 1.06 equiv) and 4-chlorobenzenethiol ( $0.1369 \mathrm{~g}, 0.947 \mathrm{mmol}, 1$ equiv). The compound was obtained as a pale-yellow solid $\left(0.2243 \mathrm{~g}, 74 \%\right.$ yield). ${ }^{1} \mathrm{H}$ NMR (300 MHz, Acetone- $\left.d_{6}\right)$ : $\delta 7.62-7.59(\mathrm{~m}, 2 \mathrm{H}), 7.47-7.44(\mathrm{~m}, 2 \mathrm{H}), 7.40-7.28(\mathrm{~m}, 5 \mathrm{H}), 5.55(\mathrm{~s}, 1 \mathrm{H}), 3.59(\mathrm{q}, J=5.2 \mathrm{~Hz}$, $4 \mathrm{H}), 2.67(\mathrm{q}, J=5.1 \mathrm{~Hz}, 4 \mathrm{H}) \mathrm{ppm} .{ }^{13} \mathrm{C} \mathrm{NMR}\left(75 \mathrm{MHz}\right.$, Acetone- $\left.d_{6}\right): \delta 139.4,136.6,134.3$, $133.0,129.8,129.6,129.0,128.8,83.1,67.3,50.3$ ppm. m.p. $=87-88^{\circ} \mathrm{C}$. HRMS $(\mathrm{m} / \mathrm{z})$ calcd for $\mathrm{C}_{17} \mathrm{H}_{18} \mathrm{ClNOS}\left([\mathrm{M}+\mathrm{H}]^{+}\right)$320.08704, found 320.09261 .

4-(((3,5-dichlorophenyl)thio)(phenyl)methyl)morpholine (15). Prepared according to general procedure using benzaldehyde $(0.1039 \mathrm{~g}, 0.979 \mathrm{mmol}, 1$ equiv), morpholine $(0.0891$ g, $1.02 \mathrm{mmol}, 1.04$ equiv) and 3,5-dichlorobenzenethiol ( $0.1751 \mathrm{~g}, 0.978 \mathrm{mmol}, 1 \mathrm{equiv})$. The compound was obtained as a pale-yellow solid $\left(0.2548 \mathrm{~g}, 91 \%\right.$ yield). ${ }^{1} \mathrm{H}$ NMR (300 $\mathrm{MHz}$, Acetone- $\left.d_{6}\right): \delta 7.62-7.60(\mathrm{~m}, 2 \mathrm{H}), 7.42-7.29(\mathrm{~m}, 6 \mathrm{H}), 5.80(\mathrm{~s}, 1 \mathrm{H}), 3.61-3.58(\mathrm{~m}, 4 \mathrm{H})$, 2.72-2.68 (m, 4H) ppm. ${ }^{13} \mathrm{C}$ NMR $\left(75 \mathrm{MHz}\right.$, Acetone- $\left.d_{6}\right): \delta 140.2,138.6,135.5,130.0,129.6$, 129.3, 129.1, 129.0, 126.9, 82.2, 67.3, 50.1 ppm. M.p. $=97-100{ }^{\circ} \mathrm{C}$. HRMS $(\mathrm{m} / \mathrm{z})$ calcd for $\mathrm{C}_{17} \mathrm{H}_{17} \mathrm{Cl}_{2} \mathrm{NOS}\left([\mathrm{M}+\mathrm{H}]^{+}\right)$354.04807, found 354.05168.

4-((benzylthio)(phenyl)methyl)morpholine (16). Prepared according to general procedure using benzaldehyde $(0.1025 \mathrm{~g}, 0.966 \mathrm{mmol}, 1$ equiv $)$, morpholine $(0.0865 \mathrm{~g}, 0.993 \mathrm{mmol}$, 1.03 equiv) and phenylmethanethiol ( $0.1208 \mathrm{~g}, 0.973 \mathrm{mmol}, 1.01 \mathrm{equiv})$. The compound was obtained as a pale-orange oil $(0.2278 \mathrm{~g}, 79 \%$ yield $) .{ }^{1} \mathrm{H}$ NMR (300 MHz, Acetone- $\left.d_{6}\right): \delta$ $7.41(\mathrm{~m}, 2 \mathrm{H}), 7.38-7.26(\mathrm{~m}, 8 \mathrm{H}), 4.85(\mathrm{~s}, 1 \mathrm{H}), 3.90(\mathrm{~d}, J=13.1 \mathrm{~Hz}, 1 \mathrm{H}), 3.71(\mathrm{~d}, J=13.1 \mathrm{~Hz}$, $1 \mathrm{H}), 3.61(\mathrm{t}, J=4.7 \mathrm{~Hz}, 4 \mathrm{H}), 2.64-2.57(\mathrm{~m}, 2 \mathrm{H}), 2.53-2.46(\mathrm{~m}, 2 \mathrm{H}) \mathrm{ppm} .{ }^{13} \mathrm{C} \mathrm{NMR}(101 \mathrm{MHz}$, Acetone- $\left.d_{6}\right): \delta 139.8,138.3,129.9,129.5,129.2,128.4,128.6,127.6,75.8,67.5,50.0,36.0$ ppm. HRMS $(m / z)$ calcd for $\mathrm{C}_{18} \mathrm{H}_{21} \mathrm{NOS}\left([\mathrm{M}+\mathrm{H}]^{+}\right) 300.14166$, found 300.14211 .

4-((phenethylthio)(phenyl)methyl)morpholine (17). Prepared according to general procedure using benzaldehyde $(0.1017 \mathrm{~g}, 0.958 \mathrm{mmol}, 1$ equiv $)$, morpholine $(0.0923 \mathrm{~g}, 1.06 \mathrm{mmol}$, 1.10 equiv) and 2-phenylethane-1-thiol ( $0.1315 \mathrm{~g}, 0.951 \mathrm{mmol}, 1$ equiv). The compound was obtained as a pale-yellow oil $(0.2697 \mathrm{~g}, 90 \%$ yield $) .{ }^{1} \mathrm{H}$ NMR $\left(300 \mathrm{MHz}\right.$, Acetone- $\left.d_{6}\right): \delta$ 7.48-7.45 (m, 2H), 7.38-7.22 (m, 8H), $4.97(\mathrm{~s}, 1 \mathrm{H}), 3.60(\mathrm{t}, J=4.7 \mathrm{~Hz}, 4 \mathrm{H}), 2.90-2.88(\mathrm{~m}, 3 \mathrm{H})$, 2.77-2.73 (m, 1H), 2.64-2.50 (m, 4H) ppm. ${ }^{13} \mathrm{C}$ NMR (101 MHz, Acetone- $\left.d_{6}\right): \delta 141.8,139.0$, $129.5,129.4,129.2,129.1,128.8,128.5,127.0,76.8,67.5,67.3,50.2,37.3,33.6$ ppm. HRMS $(\mathrm{m} / z)$ calcd for $\mathrm{C}_{19} \mathrm{H}_{23} \mathrm{NOS}\left([\mathrm{M}+\mathrm{H}]^{+}\right) 314.15731$, found 314.15854 .

4-(phenyl(propylthio)methyl)morpholine (18). Prepared according to general procedure using benzaldehyde ( $0.100 \mathrm{~g}, 0942 \mathrm{mmol}, 1$ equiv), morpholine (0.082 g, $0.942 \mathrm{mmol}, 1$ equiv) and propanethiol ( $0.0718 \mathrm{~g}, 0.942 \mathrm{mmol}, 1$ equiv). The compound was obtained as a pale-yellow oil $(0.1687 \mathrm{~g}, 71 \%$ yield $) .{ }^{1} \mathrm{H}$ NMR $\left(400 \mathrm{MHz}\right.$, Acetone- $\left.d_{6}\right): \delta 7.52-7.48(\mathrm{~m}$, 2H), 7.37-7.32 (m, 2H), 7.31-7.27 (m, 1H), 4.95 (s, 1H), 3.65-3.54 (m, 6H), 2.58 (dt, J = 10.9, 
$5.7 \mathrm{~Hz}, 5 \mathrm{H}), 2.48(\mathrm{dt}, J=12.6,7.3 \mathrm{~Hz}, 2 \mathrm{H}), 1.59(\mathrm{~m}, 2 \mathrm{H}), 0.95(\mathrm{t}, J=7.3 \mathrm{~Hz}, 3 \mathrm{H}) \mathrm{ppm} .{ }^{13} \mathrm{C}$ NMR (101 MHz, Acetone- $\left.d_{6}\right): \delta 139.4,129.4,128.7,128.3,77.1,67.4,50.2,34.3,23.9,13.8$ ppm. HRMS $(\mathrm{m} / \mathrm{z})$ calcd for $\mathrm{C}_{14} \mathrm{H}_{21} \mathrm{NOS}\left([\mathrm{M}+\mathrm{H}]^{+}\right)$252.14166, found 252.13803.

4-((dodecylthio)(phenyl)methyl)morpholine (19). Prepared according to general procedure using benzaldehyde $(0.1 \mathrm{~g}, 0.942 \mathrm{mmol}, 1$ equiv), morpholine $(0.0821 \mathrm{~g}, 0.942 \mathrm{mmol}, 1$ equiv) and dodecanethiol $(0.191 \mathrm{~g}, 0.942 \mathrm{mmol}, 1$ equiv). The compound was obtained as a white solid $\left(0.3468 \mathrm{~g}, 97 \%\right.$ yield). ${ }^{1} \mathrm{H}$ NMR $\left(300 \mathrm{MHz}\right.$, Acetone- $\left.d_{6}\right): \delta 7.52-7.46(\mathrm{~m}, 2 \mathrm{H})$, 7.39-7.27 (m, 3H), $4.96(\mathrm{~s}, 1 \mathrm{H}), 3.60(\mathrm{dd}, \mathrm{J}=5.3,4.0 \mathrm{~Hz}, 5 \mathrm{H}), 2.62-2.55(\mathrm{~m}, 5 \mathrm{H}), 1.58(\mathrm{dq}, \mathrm{J}=$ 8.3, 7.0 Hz, 2H), $1.28(\mathrm{~s}, 21 \mathrm{H}), 0.90-0.85(\mathrm{~m}, 3 \mathrm{H}) \mathrm{ppm} .{ }^{13} \mathrm{C}$ NMR $\left(101 \mathrm{MHz}\right.$, Acetone- $\left.d_{6}\right)$ : 139.5, 129.5, 128.4, 77.1, 67.5, 50.3, 32.6, 32.2, 30.7, 30.4, 30.3, 30.2, 30.1, 29.9, 29.6, 23.3, 14.4 ppm. m.p. $=52-54{ }^{\circ} \mathrm{C}$. HRMS $(\mathrm{m} / z)$ calcd for $\mathrm{C}_{23} \mathrm{H}_{39} \mathrm{NOS}\left([\mathrm{M}+\mathrm{H}]^{+}\right) 378.28521$, found 378.28370 .

\subsection{General Procedure for the Thiol Exchange}

Aminal $20(0.100 \mathrm{~g}, 0.381 \mathrm{mmol})$ was dissolved in a freshly prepared solution of $\mathrm{Cu}(\mathrm{OTf})_{2}(0.00138 \mathrm{~g}, 0.01$ equiv $)$ in acetonitrile $(1.9 \mathrm{~mL})$. Thiophenol $(0.042 \mathrm{~g}, 0.381 \mathrm{mmol})$ was added and the mixture was allowed to react for $10 \mathrm{~min}$. The crude was evaporated under reduced pressure and the obtained solid was washed with cold distilled water (5 $\mathrm{mL})$, yielding thioaminal 2 as a pale-yellow solid (0.102 $\mathrm{g}, 94 \%$ yield).

\subsection{General Procedure for UV Stabilities Experiments of Thioaminals 9, 11 and $\mathbf{1 4}$}

Thioaminals were dissolved in $\mathrm{MeOH}(10 \mathrm{mM})$ and diluted in a solution of $\mathrm{MeCN}$ or ammonium acetate $20 \mathrm{mM}$ ( $\mathrm{pH}$ 7) buffer at $0.01 \mathrm{mM}$.

Then, $10 \mathrm{mM}$ solutions of aminals $\mathbf{9 , 1 1}$ and $\mathbf{1 4}$ in methanol were freshly prepared and were diluted in $2 \mathrm{~mL}$ of acetonitrile or ammonium acetate $20 \mathrm{mM}(\mathrm{pH}$ 7) buffer to a final concentration of $100 \mu \mathrm{M}$. The quartz cuvette was swiftly mixed by inversion and analyzed by UV-Visible spectroscopy. Full-scan analyses were performed every minute until a constant absorbance value was reached. To compare the results obtained, we plotted the absorbance values obtained for each thioaminal at a particular wavelength over time $(250 \mathrm{~nm})$.

\section{Conclusions}

The preparation of $N, S$-thioaminals is an ongoing challenge, due to both the reactivity and the stability of the products. Previous conditions required high temperatures and/or hazardous reagents. In accordance with the principles of green chemistry, in this work we expanded our previously reported conditions to thioaminals, affording the products under mild conditions, in aqueous media promoted by catalytic amounts of base metal copper(II) triflate. We hope that this report will contribute to the valorization of the thioaminal scaffold, both for drug discovery but also as a synthon for further derivatizations.

Supplementary Materials: The following supporting information can be downloaded online. Figures S1-S20: ${ }^{1} \mathrm{H}$ NMR, ${ }^{13} \mathrm{C}$ NMR of compounds 1-19. Figure S21: Variation of UV spectrum of thioaminal 9 after $20 \mathrm{~s}$ at pH 7; Figure S22: Variation of UV spectrum of thioaminal 11 after $20 \mathrm{~s}$ at pH 7; Figure S23: Variation of UV spectrum of thioaminal 14 after $20 \mathrm{~s}$ at $\mathrm{pH}$ 7. Reference [41] is Cited in Supplementary Materials.

Author Contributions: Conceptualization and Methodology, L.A.S.C. and R.F.A.G.; Writing—original draft, R.F.A.G. and L.A.S.C.; Writing-review and editing, L.A.S.C., R.F.A.G. and C.A.M.A.; funding acquisition, C.A.M.A. All authors have read and agreed to the published version of the manuscript.

Funding: The authors acknowledge Fundação para a Ciência e Tecnologia (FCT) for financial support (PD/BD/143127/2019, PTDC/QUI-QOR/32008/2017, UIDB/04138/2020 and UIDP/04138/2020). The project leading to this application has received funding from the European Union's Horizon 2020 research and innovation programme under grant agreement No 951996).

Institutional Review Board Statement: Not applicable. 
Informed Consent Statement: Not applicable.

Data Availability Statement: Electronic supplementary information is given, including all NMR spectra and additional experimental details regarding UV stabilities studies.

Conflicts of Interest: The authors declare no conflict of interest.

Sample Availability: Samples of the compounds are not available from the authors.

\section{References}

1. Lowe, A.B. Thiol-ene "click" reactions and recent applications in polymer and materials synthesis: A first update. Polym. Chem. 2014, 5, 4820-4870. [CrossRef]

2. Zheng, Y.; Li, S.; Weng, Z.; Gao, C. Hyperbranched polymers: Advances from synthesis to applications. Chem. Soc. Rev. 2015, 44, 4091-4130. [CrossRef] [PubMed]

3. Summonte, S.; Racaniello, G.F.; Lopedota, A.; Denora, N.; Bernkop-Schnurch, A. Thiolated polymeric hydrogels for biomedical application: Cross-linking mechanisms. J. Control. Release 2021, 330, 470-482. [CrossRef]

4. Boyd, D.A. Sulfur and Its Role In Modern Materials Science. Angew. Chem. Int. Ed. 2016, 55, 15486-15502. [CrossRef]

5. Bottecchia, C.; Noël, T. Photocatalytic Modification of Amino Acids, Peptides, and Proteins. Chem. A Eur. J. 2018, 25, 26-42. [CrossRef] [PubMed]

6. Gunnoo, S.B.; Madder, A. Chemical Protein Modification through Cysteine. Chembiochem 2016, 17, 529-553. [CrossRef] [PubMed]

7. Ravasco, J.; Faustino, H.; Trindade, A.; Gois, P.M.P. Bioconjugation with Maleimides: A Useful Tool for Chemical Biology. Chemistry 2019, 25, 43-59. [CrossRef]

8. Ilardi, E.A.; Vitaku, E.; Njardarson, J.T. Data-mining for sulfur and fluorine: An evaluation of pharmaceuticals to reveal opportunities for drug design and discovery. J. Med. Chem. 2014, 57, 2832-2842. [CrossRef] [PubMed]

9. Feng, M.; Tang, B.; Liang, S.H.; Jiang, X. Sulfur Containing Scaffolds in Drugs: Synthesis and Application in Medicinal Chemistry. Curr. Top. Med. Chem. 2016, 16, 1200-1216. [CrossRef] [PubMed]

10. Beno, B.R.; Yeung, K.S.; Bartberger, M.D.; Pennington, L.D.; Meanwell, N.A. A Survey of the Role of Noncovalent Sulfur Interactions in Drug Design. J. Med. Chem. 2015, 58, 4383-4438. [CrossRef] [PubMed]

11. Kaur Manjal, S.; Kaur, R.; Bhatia, R.; Kumar, K.; Singh, V.; Shankar, R.; Kaur, R.; Rawal, R.K. Synthetic and medicinal perspective of thiazolidinones: A review. Bioorg. Chem. 2017, 75, 406-423. [CrossRef] [PubMed]

12. Jain, V.S.; Vora, D.K.; Ramaa, C.S. Thiazolidine-2,4-diones: Progress towards multifarious applications. Bioorg. Med. Chem. 2013, 21, 1599-1620. [CrossRef]

13. Pathania, S.; Narang, R.K.; Rawal, R.K. Role of sulphur-heterocycles in medicinal chemistry: An update. Eur. J. Med. Chem. 2019, 180, 486-508. [CrossRef]

14. Biswas, S.; Kubota, K.; Orlandi, M.; Turberg, M.; Miles, D.H.; Sigman, M.S.; Toste, F.D. Enantioselective Synthesis of N,S-Acetals by an Oxidative Pummerer-Type Transformation using Phase-Transfer Catalysis. Angew. Chem. Int. Ed. Engl. 2018, 57, 589-593. [CrossRef] [PubMed]

15. Ramachandran, G.; Karthikeyan, N.S.; Giridharan, P.; Sathiyanarayanan, K.I. Efficient iodine catalyzed three components domino reaction for the synthesis of 1-((phenylthio)(phenyl)methyl)pyrrolidin-2-one derivatives possessing anticancer activities. Org. Biomol. Chem. 2012, 10, 5343-5346. [CrossRef] [PubMed]

16. Ingle, G.K.; Mormino, M.G.; Wojtas, L.; Antilla, J.C. Chiral Phosphoric Acid-Catalyzed Addition of Thiols to N-Acyl Imines: Access to Chiral N,S-Acetals. Org. Lett. 2011, 13, 4822-4825. [CrossRef] [PubMed]

17. Chikashita, H.; Komazawa, S.I.; Ishimoto, N.; Inoue, K.; Itoh, K. Nonacidic and Highly Chemoselective Protection of the Carbonyl Function. 3-Methylbenzothiazolines as a Base- and Acid.Resistant Protected Form for the Carbonyl Groyups. Bull. Chem. Soc. Jpn. 1989, 62, 1215-1225. [CrossRef]

18. JVoß, J.; Wiegand, G.; Hülsmeyer, K. Elektroreduktion organischer Verbindungen. Chem. Ber. 1985, 118, $4806-4820$.

19. Ratner, S.; Clarke, H.T. The Action of Formaldehyde upon Cysteine. J. Am. Chem. Soc. 1937, 59, 200-206. [CrossRef]

20. Dey, A.; Hajra, A. Metal-Free Synthesis of 2-Arylbenzothiazoles from Aldehydes, Amines, and Thiocyanate. Org. Lett. 2019, 21, 1686-1689. [CrossRef] [PubMed]

21. Alper, K.K.A.H. Novel, Metal-Catalyzed Carbonylation of Acyclic Organic. Compounds. The Regiospecific Carbonylation of N,S-Acetals. J. Org. Chem. 1994, 59, 1414-1417.

22. Bolognese, A.; Correale, G.; Manfra, M.; Lavecchia, A.; Novellino, E.; Barone, V. Thiazolidin-4-one formation. Mechanistic and synthetic aspects of the reaction of imines and mercaptoacetic acid under microwave and conventional heating. Org. Biomol. Chem. 2004, 2, 2809-2813. [CrossRef] [PubMed]

23. Alan, R.; Katritzky, M.S.; Bayyuk, S. The preparation of some N- and S-acetals of benzaldehyde and terephtaldehyde. Synthesis 1986, 10, 804-807.

24. Sheldon, R.A. Fundamentals of green chemistry: Efficiency in reaction design. Chem. Soc. Rev. 2012, 41, 1437-1451. [CrossRef]

25. Sheldon, R.A. Green and sustainable manufacture of chemicals from biomass: State of the art. Green Chem. 2014, 16, 950-963. [CrossRef] 
26. Bozell, J.J.; Petersen, G.R. Technology development for the production of biobased products from biorefinery carbohydrates-the US Department of Energy's "Top 10" revisited. Green Chem. 2010, 12, 539-554. [CrossRef]

27. Werpy, T.A.; Holladay, J.; White, J.F. Top Value Added Chemicals from Biomass. Volume I-Results of Screening for Potential. Candidates from Sugars and Synthesis Gas. US Dep. Energy 2004. [CrossRef]

28. Zheng, X.; Liu, Y.; Wan, J.P. Metal-Free Synthesis of 1, 2, 3-Triazoles in Pure Water via the Enamine Modified Annulation Reactions with Tosyl Azide. Chin. J. Org. Chem. 2021, 41, 2700-2706. [CrossRef]

29. Casalta, C.; Gourlaouen, C.; Bouzbouz, S. Iridium(III) Catalyzed Z-Selective Allylic Arylation of alpha-Fluoro But-1-enoic Acid Amides via beta-F-Elimination in Water. Org. Lett. 2021, 23, 8122-8126. [CrossRef] [PubMed]

30. Peramo, A.; Abdellah, I.; Pecnard, S.; Mougin, J.; Martini, C.; Couvreur, P.; Huc, V.; Desmaële, D.J.M. A self-assembling NHC-Pd-loaded calixarene as a potent catalyst for the Suzuki-Miyaura cross-coupling reaction in water. Molecules 2020, 25, 1459. [CrossRef]

31. Gan, L.; Wei, L.; Wan, J.P.J.C. Catalyst-Free Synthesis of $\alpha$-Diazoketones in Water by Microwave Promoted Enaminone C= C Double Bond Cleavage. ChemistrySelect 2020, 5, 7822-7825. [CrossRef]

32. Fu, L.; Cao, X.; Wan, J.P.; Liu, Y. Synthesis of Enaminone-Pd (II) Complexes and Their Application in Catalysing Aqueous Suzuki-Miyaura Cross Coupling Reaction. Chin. J. Chem. 2020, 38, 254-258. [CrossRef]

33. Gilcher, E.B.; Chang, H.; Huber, G.W.; Dumesic, J.A. Controlled hydrogenation of a biomass-derived platform chemical formed by aldol-condensation of 5-hydroxymethyl furfural (HMF) and acetone over Ru, Pd, and Cu catalysts. Green Chem. 2022. [CrossRef]

34. Gomes, R.F.A.; Coelho, J.A.S.; Afonso, C.A.M. Direct Conversion of Activated 5-Hydroxymethylfurfural into delta-Lactone-Fused Cyclopentenones. ChemSusChem 2019, 12, 420-425. [CrossRef] [PubMed]

35. Gomes, R.F.A.; Mitrev, Y.N.; Simeonov, S.P.; Afonso, C.A.M. Going Beyond the Limits of the Biorenewable Platform: Sodium Dithionite-Promoted Stabilization of 5-Hydroxymethylfurfural. ChemSusChem 2018, 11, 1612-1616. [CrossRef] [PubMed]

36. Gomes, R.F.A.; Esteves, N.R.; Coelho, J.A.S.; Afonso, C.A.M. Copper(II) Triflate As a Reusable Catalyst for the Synthesis of trans-4,5-Diamino-cyclopent-2-enones in Water. J. Org. Chem. 2018, 83, 7509-7513. [CrossRef] [PubMed]

37. Pereira, J.G.; António, J.P.M.; Mendonça, R.; Gomes, R.F.A.; Afonso, C.A.M. Rediscovering aminal chemistry: Copper(ii) catalysed formation under mild conditions. Green Chem. 2020, 22, 7484-7490. [CrossRef]

38. Nardi, M.; Costanzo, P.; De Nino, A.; Di Gioia, M.L.; Olivito, F.; Sindona, G.; Procopio, A. Water excellent solvent for the synthesis of bifunctionalized cyclopentenones from furfural. Green Chem. 2017, 19, 5403-5411. [CrossRef]

39. Alan, R.; Katritzky, W.-Q.F.; Long, Q.-H. A Convenient Synthesis of N-(alpha-Alkoxyalkyl)- and N-[alpha-(Alkylthio)alkyl]amines. Synthesis 1992, 1993, 229-232.

40. Sakai, H.; Ito, K.; Sekiya, M. Reaction of N-(Dialkylaminomethyl)amides and N-(alpha-Dialkylaminobenzyl)amides with Sulfides and Cyanide. Chem. Pharm. Bull. 1973, 21, 2257-2264. [CrossRef]

41. Fawwaz, M.; Mishiro, K.; Nishii, R.; Sawazaki, I.; Shiba, K.; Kinuya, S.; Ogawa, K. Synthesis and Fundamental Evaluation of Radioiodinated Rociletinib (CO-1686) as a Probe to Lung Cancer with L858R/T790M Mutations of Epidermal Growth Factor Receptor (EGFR). Molecules 2020, 25, 2914. [CrossRef] [PubMed] 